\title{
Melanoma in mice casts doubt on scarcity of cancer stem cells
}

The rare 'cancer stem cells' thought to seed cancer growth are not so rare after all, according to researchers in Michigan. If they're right, then the strategies used for some cancer therapies may need to change.

The research challenges the notion that there is an elite set of tumour cells that continually regenerates a cancer, says William Kaelin, who studies tumour-suppressor proteins at the Dana-Farber Cancer Institute in Boston, Massachusetts, and was not affiliated with the study. "If it turns out that the cells were never rare, then we've been studying those cells all along," he says. "And a cancer stem cell is just a cancer cell."

Previous studies had transplanted human cancer cells into immunocompromised mice that still had enough of an immune system to fight the transplanted cells. The latest work introduced human melanoma cells into a more severely immunocompromised mouse strain ${ }^{1,2}$. Under these conditions, the rate of tumour initiation surged. In less immunocompromised mice, only 1 in 837,000 cells regenerated melanoma. In the new mice 1 in 4 cells did.

Sean Morrison, who led the work at the University of Michigan in Ann Arbor, warns researchers to interpret his results with caution. "There

"To say this invalidates the field is an overinterpretation." are some people out there who look at the paper and say that this completely invalidates the cancer stem-cell field," he says. "That's an overinterpretation." He argues that the evidence for cancer stem cells in certain cancers, such as leukaemia, is particularly convincing.

But researchers who report cancer stem cells in solid tumours should be wary of their results, he says, and should repeat their experiments using techniques that could help transplanted human cells survive in mice. Otherwise, it's unclear whether they have detected a rare subset of cells that can propagate tumours - or simply a rare subset of human cells that can establish themselves in mice.

Cancer stem cells were first identified in leukaemia $^{3}$ in 1994, by John Dick of the University of Toronto, Canada. Since then, they've been reported in various cancers including those of the brain, breast, colon and pancreas. Researchers agree that techniques used to identify cancer stem cells - sorting cells from a human cancer and transplanting them into mice - are not ideal. Cells that might initiate tumours in patients might not show up in mouse-based assays.
In fact, the rarity of cancer stem cells was called into question last year ${ }^{4}$ by a team of researchers led by Andreas Strasser at the Walter and Eliza Hall Institute of Medical Research in Melbourne, Australia. In mice engineered to overexpress an oncogene, the team showed that leukaemia cells readily established cancers when transplanted into other mice. But other scientists questioned whether those results would apply to spontaneously occurring cancers, human cancers and solid tumours.

Some proponents of the cancer stem-cell hypothesis say they wonder whether melanoma, as studied by Morrison, is unusual in that it might have a particularly high frequency of tumourinitiating cells or an increased likelihood of being cleared out by the immune system. Most importantly, these researchers say that self-renewing cells don't need to be rare to be worthy of special attention. After all, most cancer tumours contain a mixture of cells, and it is still possible that a subset of tumour cells could generate the other cells. "We need to figure out the different functions of the cells in the cancer," says Peter Dirks of the Hospital for Sick Children in Toronto, who has identified cancer stem cells in the brain.

The work has implications for targeting cancer therapies - in particular, whether to develop drugs that go after cancer cells' ability to proliferate rapidly, or to attack the cells seeding the tumours. Michael Clarke, a stem-cell biologist at Stanford University in Palo Alto, California, says that current therapies work to block proliferation. Some experimental therapies that block stemcell pathways are in clinical trials, such as those being developed by Genentech in South San Francisco, California, and Merck of Whitehouse Station, New Jersey. Clarke and others think it is essential to identify and purify cancer stem cells to work out the best pathways to target.

Other researchers, such as Strasser, see little use in chasing special subsets. In those cases where the cancer doesn't return, he says, "it's where we are very good at killing all the cells of the tumour".

\section{Monya Baker}

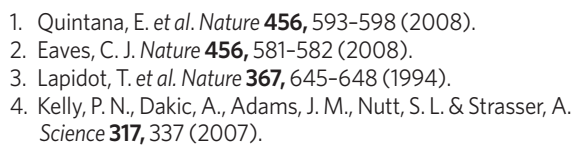

1. Quintana, E. et al. Nature 456, 593-598 (2008).

2. Eaves, C. J. Nature 456, 581-582 (2008).

3. Lapidot, T. et al. Nature 367, 645-648 (1994).

4. Kelly, P. N., Dakic, A., Adams, J. M., Nutt, S. L. \& Strasser, A. Science 317, 337 (2007).

See also Authors, page xiii. 